International Journal of Difference Equations (IJDE).

ISSN 0973-6069, Volume 16, Number 1, (2021). 59-79

(C) Research India Publications

https://dx.doi.org/10.37622/IJDE/16.1.2021.59-79

\title{
A Comparative Study of Approximate Solution of Fifth-order Boundary-value Problems by Applying Haar Wavelets Technique and Galerkin Method with Quartic B-splines
}

\author{
Kailash Yadav \\ Hindustan Institute of Technology and Science (HITS), \\ Chennai, Tamilnadu, India.
}

\begin{abstract}
Wavelet analysis is well developed mathematical tool which can be also applied in numerical analysis. Here application of the Haar wavelet techniques has been discussed for finding numerical solution of fifth-order boundary value problems. Accuracy of the considered technique is demonstrated by means of three numerical examples, taken from well existing research article.
\end{abstract}

Mathematics Subject Classification (2000). 65L10, 65T60.

Keywords and Phrases. Boundary value problem, Haar wavelet, Quartic B-spline, collocation point, grid point.

\section{INTRODUCTION}

In this paper, we consider a general fifth-order linear boundary value problem (BVP) given by

$$
\begin{aligned}
& y^{(5)}(x)+A(x) y^{(4)}(x)+B(x) y^{\prime \prime \prime}(x) \\
+ & C(x) y^{\prime \prime}(x)+D(x) y^{\prime}(x)+E(x) y(x)=f(x), x \in[a, b],
\end{aligned}
$$

with boundary conditions

$$
y(a)=p, y(b)=q, y^{\prime}(a)=r, y^{\prime}(b)=s, y^{\prime \prime}(a)=t,
$$

where $y$ and $f$ are continuous functions defined in the interval $x \in[a, b], f \in C^{5}[a, b]$ 
is real and $p, q, r, s$ and $t$ are finite real constants. Generally, this type of fifth order boundary value problems(BVPs) arises in the mathematical modeling of viscoelastic flows and other branches of mathematical, physical, and engineering sciences (one can see [1]-[8]). Theorems, which list the conditions for the existence and uniqueness of the solution of fifth-order BVPs are discussed in [9]. In the present years wavelet viewpoint is becoming more interest in the field of numerical approximations. Different type of wavelets and approximating functions have been used for this motive in the article ([10]-[12]) . The objective of this contribution is to construct a simplified collocation method with the Haar basis function for the numerical solution of linear BVPs arising in the mathematical modeling of the different engineering applications. The main idea of this technique is to convert a differential equation into algebraic one. In order to approximate the solution of differential equation, we collocate the algebraic equations at collocation points. The benefits of Haar wavelets transform are sparse matrix of representations than other existing method.

Let us defined $M=2^{J}$, where $J$ is the maximal level of resolution. Next two parameters are introduced: the dilatation parameter $j=0,1, \ldots, J$ and translation parameter $k=0,1, \ldots, m-1$ (here the notation $m=2^{j}$ is introduced). The Haar wavelet family for $x \in[0,1)$ defined as ([13]-[16]):

$$
h_{i}(x)= \begin{cases}1 & \text { for } x \in\left[\xi_{1}, \xi_{2}\right) \\ -1 & \text { for } x \in\left[\xi_{2}, \xi_{3}\right) \\ 0 & \text { elsewhere }\end{cases}
$$

where

$$
\xi_{1}=\frac{k}{m}, \xi_{2}=\frac{k+0.5}{m}, \xi_{3}=\frac{k+1}{m} .
$$

The index $i$ in (3), is calculated using the formula $i=2^{j}+k+1$. In case of minimal values $m=1, k=0$, we have $i=2$. The maximal value of $i$ is $i=2 M=2^{J+1}$. The case $i=1$, corresponding to the scaling function

$$
h_{1}(x)= \begin{cases}1 & \text { for } x \in[0,1) \\ 0 & \text { elsewhere }\end{cases}
$$

If the maximal level of resolution $J$ is prescribed then it follows from (3) that

$$
\int_{0}^{1} h_{i}(x) h_{l}(x) d x= \begin{cases}2^{-j} & \text { for } l=i \\ 0 & \text { for } l \neq i\end{cases}
$$


So we see that the Haar wavelets are orthogonal to each other. In the following, we need the integrals of Haar functions

$$
\underbrace{\int_{0}^{x} \int_{0}^{x} \ldots \int_{0}^{x}}_{(v-\text { times })} h_{i}(t)(d t)^{v}=\frac{1}{(v-1) !} \int_{0}^{1}(x-t)^{v-1} h_{i}(t) d t, v=1,2, \ldots
$$

Taking account of (3), these integrals can be calculated analytically; by doing it first five of them are given by

$$
\begin{aligned}
& p_{i, 1}(x)= \begin{cases}0 & \text { for } x \in\left[0, \xi_{1}\right), \\
x-\xi_{1} & \text { for } x \in\left[\xi_{1}, \xi_{2}\right), \\
\xi_{3}-x & \text { for } x \in\left[\xi_{2}, \xi_{3}\right),\end{cases} \\
& p_{i, 2}(x)= \begin{cases}0 & \text { for } x \in\left[0, \xi_{1}\right), \\
\frac{1}{2}\left(x-\xi_{1}\right)^{2} & \text { for } x \in\left[\xi_{1}, \xi_{2}\right), \\
\frac{1}{4 m^{2}}-\frac{1}{2}\left(\xi_{3}-x\right)^{2} & \text { for } x \in\left[\xi_{2}, \xi_{3}\right), \\
\frac{1}{4 m^{2}} & \text { for } x \in\left[\xi_{3}, 1\right),\end{cases} \\
& p_{i, 3}(x)= \begin{cases}0 & \text { for } x \in\left[0, \xi_{1}\right), \\
\frac{1}{6}\left(x-\xi_{1}\right)^{3} & \text { for } x \in\left[\xi_{1}, \xi_{2}\right), \\
\frac{1}{4 m^{2}}\left(x-\xi_{2}\right)+\frac{1}{6}\left(\xi_{3}-x\right)^{3} & \text { for } x \in\left[\xi_{2}, \xi_{3}\right), \\
\frac{1}{4 m^{2}}\left(x-\xi_{2}\right) & \text { for } x \in\left[\xi_{2}, 1\right),\end{cases} \\
& p_{i, 4}(x)= \begin{cases}0 & \text { for } x \in\left[0, \xi_{1}\right), \\
\frac{1}{24}\left(x-\xi_{1}\right)^{4} & \text { for } x \in\left[\xi_{1}, \xi_{2}\right), \\
\frac{1}{8 m^{2}}\left(x-\xi_{2}\right)^{2}-\frac{1}{24}\left(\xi_{3}-x\right)^{4}+\frac{1}{192 m^{4}} & \text { for } x \in\left[\xi_{2}, \xi_{3}\right), \\
\frac{1}{8 m^{2}}\left(x-\xi_{2}\right)^{2}+\frac{1}{192 m^{4}} & \text { for } x \in\left[\xi_{2}, 1\right),\end{cases} \\
& p_{i, 5}(x)= \begin{cases}0 & \text { for } x \in\left[0, \xi_{1}\right), \\
\frac{1}{120}\left(x-\xi_{1}\right)^{5} & \text { for } x \in\left[\xi_{1}, \xi_{2}\right), \\
\frac{1}{24 m^{2}}\left(x-\xi_{2}\right)^{3}+\frac{1}{120}\left(\xi_{3}-x\right)^{5}+\frac{1}{192 m^{4}}\left(x-\xi_{2}\right) & \text { for } x \in\left[\xi_{2}, \xi_{3}\right), \\
\frac{1}{24 m^{2}}\left(x-\xi_{2}\right)^{3}+\frac{1}{192 m^{4}}\left(x-\xi_{3}\right)+\frac{1}{384 m^{5}} & \text { for } x \in\left[\xi_{2}, 1\right) .\end{cases}
\end{aligned}
$$

We also introduce the following notation:

$$
C_{i, v}=\int_{0}^{1} p_{i, v}(x) d x, v=1,2, \ldots
$$


Any function $f(x)$ which is square integrable in the interval $(0,1)$ can be expressed as an infinite sum of Haar wavelets in the form :

$$
f(x)=\sum_{i=1}^{\infty} a_{i} h_{i}(x) .
$$

The above series terminates at finite terms if $f(x)$ is piecewise constant or can be approximated as piecewise constant during each subinterval.

The rest of the paper is organized as follows : Section 2, general formulation of the numerical technique based on Haar wavelets. Just before final section, we consider three numerical problems for comparison with existing methods. Finally, in the last section, we give the concluding remarks.

\section{HAAR WAVELET METHOD FOR SOLVING FIFTH ORDER DIFFERENTIAL EQUATIONS}

To apply Haar wavelet method for problem (1) we approximate highest order derivative $y^{(5)}(x)$ using Haar wavelet series as follows

$$
y^{(5)}(x)=\sum_{i=1}^{2 M} a_{i} h_{i}(x) .
$$

Taking integration (15) and using the boundary conditions (2) with $a=0, b=1$, we can get $y^{(4)}(x), y^{\prime \prime \prime}(x), y^{\prime \prime}(x), y^{\prime}(x)$ and $y(x)$ as :

$$
\begin{gathered}
y^{(4)}(x)=y^{(4)}(0)+\sum_{i=1}^{2 M} a_{i} p_{i, 1}(x), \\
y^{\prime \prime \prime}(x)=y^{\prime \prime \prime}(0)+x y^{(4)}(0)+\sum_{i=1}^{2 M} a_{i} p_{i, 2}(x), \\
y^{\prime \prime}(x)=t+x y^{\prime \prime \prime}(0)+\frac{x^{2}}{2} y^{(4)}(0)+\sum_{i=1}^{2 M} a_{i} p_{i, 3}(x), \\
y^{\prime}(x)=r+t x+\frac{x^{2}}{2} y^{\prime \prime \prime}(0)+\frac{x^{3}}{6} y^{(4)}(0)+\sum_{i=1}^{2 M} a_{i} p_{i, 4}(x), \\
y(x)=p+r x+t \frac{x^{2}}{2}+\frac{x^{3}}{6} y^{\prime \prime \prime}(0)+\frac{x^{4}}{24} y^{(4)}(0)+\sum_{i=1}^{2 M} a_{i} p_{i, 5}(x),
\end{gathered}
$$

where $p_{i, 1}, p_{i, 2}, p_{i, 3}, p_{i, 4}$ and $p_{i, 5}$ are defined in equations (8), (9), (10), (11) and (12), respectively. The presence of two integration constants permit us the additional of 
two more equations which can be done by using particulars on the above equation and boundary conditions at both ends of the rule. Discretization using collocation points. $x_{j}=\frac{j-0.5}{2 M}, j=1,2, \ldots, 2 M$ of the equations $(15)-(20)$ can be reduced into the following matrix form

$$
\begin{aligned}
& y^{(5)}(x)=\left[\begin{array}{ccccc}
h_{1}\left(x_{1}\right) & \ldots & h_{2 M}\left(x_{1}\right) & 0 & 0 \\
h_{1}\left(x_{2}\right) & \ldots & h_{2 M}\left(x_{2}\right) & 0 & 0 \\
\ldots & & \ldots & \ldots & \ldots \\
\ldots & \ldots & \ldots & \ldots \\
\ldots & & \ldots & \ldots & \ldots \\
\ldots & \ldots & \ldots & \ldots \\
\ldots & & \ldots & \ldots & \ldots \\
h_{1}\left(x_{2 M}\right) & \ldots & h_{2 M}\left(x_{2 M}\right) & 0 & 0
\end{array}\right]\left[\begin{array}{c}
a_{1} \\
a_{2} \\
\cdot \\
\cdot \\
\cdot \\
a_{2 M} \\
y^{(3)}(0) \\
y^{(4)}(0)
\end{array}\right] \\
& y^{(4)}(x)=\left[\begin{array}{ccccc}
p_{1,1}\left(x_{1}\right) & \ldots & p_{2 M, 1}\left(x_{1}\right) & 0 & 1 \\
p_{1,1}\left(x_{2}\right) & \ldots & p_{2 M, 1}\left(x_{2}\right) & 0 & 1 \\
\ldots & & \ldots & \ldots & \ldots \\
\ldots & & \ldots & \ldots & \ldots \\
\ldots & & \ldots & \ldots & \ldots \\
\ldots & & \ldots & \ldots & \ldots \\
\ldots & & \ldots & \ldots & \ldots \\
p_{1,1}\left(x_{2 M}\right) & \ldots & p_{2 M, 1}\left(x_{2 M}\right) & 0 & 1
\end{array}\right]\left[\begin{array}{c}
a_{1} \\
a_{2} \\
\cdot \\
\cdot \\
\cdot \\
a_{2 M} \\
y^{(3)}(0) \\
y^{(4)}(0)
\end{array}\right], \\
& y^{\prime \prime \prime}(x)=\left[\begin{array}{ccccc}
p_{1,2}\left(x_{1}\right) & \ldots & p_{2 M, 2}\left(x_{1}\right) & 1 & x_{1} \\
p_{1,2}\left(x_{2}\right) & \ldots & p_{2 M, 2}\left(x_{2}\right) & 1 & x_{2} \\
\ldots & & \ldots & \ldots & \ldots \\
\ldots & & \ldots & \ldots & \ldots \\
\ldots & & \ldots & \ldots & \ldots \\
\ldots & \ldots & \ldots & \ldots \\
\ldots & & \ldots & \ldots & \ldots \\
p_{1,2}\left(x_{2 M}\right) & \ldots & p_{2 M, 2}\left(x_{2 M}\right) & 1 & x_{2 M}
\end{array}\right]\left[\begin{array}{c}
a_{1} \\
a_{2} \\
\cdot \\
\cdot \\
\cdot \\
a_{2 M} \\
y^{(3)}(0) \\
y^{(4)}(0)
\end{array}\right], \\
& y^{\prime \prime}(x)=\left[\begin{array}{ccccc}
p_{1,3}\left(x_{1}\right) & \ldots & p_{2 M, 3}\left(x_{1}\right) & x_{1} & x_{1}^{2} / 2 \\
p_{1,3}\left(x_{2}\right) & \ldots & p_{2 M, 3}\left(x_{2}\right) & x_{2} & x_{2}^{2} / 2 \\
\ldots & & \ldots & \ldots & \ldots \\
\ldots & & \ldots & \ldots & \ldots \\
\ldots & & \ldots & \ldots & \ldots \\
\ldots & \ldots & \ldots & \ldots \\
\ldots & & \ldots & \ldots & \ldots \\
p_{1,3}\left(x_{2 M}\right) & \ldots & p_{2 M, 3}\left(x_{2 M}\right) & x_{2 M} & x_{2 M}^{2} / 2
\end{array}\right]\left[\begin{array}{c}
a_{1} \\
a_{2} \\
\cdot \\
\cdot \\
\cdot \\
a_{2 M} \\
y^{(3)}(0) \\
y^{(4)}(0)
\end{array}\right]+\left[\begin{array}{c}
t \\
t \\
\cdot \\
\cdot \\
\cdot \\
\cdot \\
t
\end{array}\right]
\end{aligned}
$$




$$
\begin{aligned}
& y^{\prime}(x)=\left[\begin{array}{ccccc}
p_{1,4}\left(x_{1}\right) & \ldots & p_{2 M, 4}\left(x_{1}\right) & x_{1}^{2} / 2 & x_{1}^{3} / 6 \\
p_{1,4}\left(x_{2}\right) & \ldots & p_{2 M, 4}\left(x_{2}\right) & x_{2}^{2} / 2 & x_{2}^{3} / 6 \\
\ldots & & \ldots & \ldots & \ldots \\
\ldots & \ldots & \ldots & \ldots \\
\ldots & \ldots & \ldots & \ldots \\
\ldots & \ldots & \ldots & \ldots \\
\ldots & & \ldots & \ldots & \ldots \\
p_{1,4}\left(x_{2 M}\right) & \ldots & p_{2 M, 4}\left(x_{2 M}\right) & x_{2 M}^{2} / 2 & x_{2 M}^{3} / 6
\end{array}\right]\left[\begin{array}{c}
a_{1} \\
a_{2} \\
\cdot \\
\cdot \\
\cdot \\
a_{2 M} \\
y^{(3)}(0) \\
y^{(4)}(0)
\end{array}\right] \\
& +\left[\begin{array}{c}
r+t x_{1} \\
r+t x_{2} \\
\cdot \\
\cdot \\
\cdot \\
\cdot \\
r+t x_{2 M}
\end{array}\right]
\end{aligned}
$$

$y(x)=\left[\begin{array}{ccccc}p_{1,5}\left(x_{1}\right) & \ldots & p_{2 M, 5}\left(x_{1}\right) & x_{1}^{3} / 6 & x_{1}^{4} / 24 \\ p_{1,5}\left(x_{2}\right) & \ldots & p_{2 M, 5}\left(x_{2}\right) & x_{2}^{3} / 6 & x_{2}^{4} / 24 \\ \ldots & & \ldots & \ldots & \ldots \\ \ldots & \ldots & \ldots & \ldots \\ \ldots & & \ldots & \ldots & \ldots \\ \ldots & \ldots & \ldots & \ldots \\ \ldots & & \ldots & \ldots & \ldots \\ p_{1,5}\left(x_{2 M}\right) & \ldots & p_{2 M, 5}\left(x_{2 M}\right) & x_{2 M}^{3} / 6 & x_{2 M}^{4} / 24\end{array}\right]\left[\begin{array}{c}a_{1} \\ a_{2} \\ \cdot \\ \cdot \\ \cdot \\ a_{2 M} \\ y^{(3)}(0) \\ y^{(4)}(0)\end{array}\right]$

$$
+\left[\begin{array}{c}
p+r x_{1}+t x_{1}^{2} / 2 \\
p+r x_{2}+t x_{2}^{2} / 2 \\
\cdot \\
\cdot \\
\cdot \\
\cdot \\
p+r x_{2 M}+t x_{2 M}^{2} / 2
\end{array}\right]
$$


The value of unknown term $y^{(4)}(0)$ and $y^{\prime \prime \prime}(0)$ be calculated by integrating equation (18) and (19) from 0 to 1 and is given by

$$
y^{\prime \prime \prime}(0)=6\left\{4 q-4 p-3 r-s-t+\sum_{i=1}^{2 M} a_{i}\left(C_{i, 3}-4 C_{i, 4}\right)\right\},
$$

and

$$
y^{(4)}(0)=6\left\{4 s+8 r+2 t-12 q+12 p+4 \sum_{i=1}^{2 M} a_{i}\left(3 C_{i, 4}-C_{i, 3}\right)\right\} .
$$

These values are substituted in the expressions (16), (17), (18), (19) and (20) in order to obtain system of equations whose and solution gives us the Haar coefficients. Babolian and Shahsavaran [17] have shown that the error bound is inversely proportional to the level of resolution of Haar wavelet. This ensures the convergence of Haar wavelet approximation when $M$ is increased.

\section{NUMERICAL EXAMPLES}

To demonstrate the applicability of the method, we consider the three linear BVPs, which have been widely discussed in the approximate and exact solutions are available for comparison. The computer characteristic is Microsoft Windows $10 \operatorname{Intel}(\mathrm{R})$ Core(TM) i3 CPU M380@ $2.53 \mathrm{GHz}$ with 3.00 GB of RAM, 64-bit operating system throughout this paper. Here we use the software MATLAB R2014a, for numerical computing.

Example 5.1: Consider the linear boundary value problem [18]:

$$
\begin{aligned}
y^{(5)}(x)+y(x)= & -4 e^{x}\left(x^{2} \cos x-2 x \cos x-9 \cos x\right) \\
& -e^{x}\left(3 x^{2} \sin x+34 x \sin x+3 \sin x\right), \\
& 0<x<1,
\end{aligned}
$$

with boundary conditions

$$
y(0)=y(1)=0, \quad y^{\prime}(0)=1, \quad y^{\prime}(1)=0, \quad y^{\prime \prime}(0)=-2 .
$$

The exact solution for the above example is

$$
y(x)=e^{x}(1-x)^{2} \sin x .
$$


Example 5.2: Consider the linear boundary value problem [18]:

$$
y^{(5)}(x)-y^{(4)}(x)=-(2 x+7) e^{x}, 0<x<1,
$$

with boundary conditions

$$
y(0)=y(1)=0, \quad y^{\prime}(0)=1, \quad y^{\prime}(1)=-e, \quad y^{\prime \prime}(0)=0 .
$$

The exact solution is given by

$$
y(x)=x(1-x) e^{x} .
$$

Example 5.3: Consider the linear boundary value problem [18]:

$$
\begin{array}{r}
y^{(5)}(x)+x y(x)=(1-x) \cos x-5 \sin x+x \sin x-x^{2} \sin x, \\
0<x<1,
\end{array}
$$

with boundary conditions

$$
\left.y(0)=y(1)=0, \quad y^{\prime}(0)=1, \quad y^{\prime}(1)=-\sin 1, \quad y^{\prime \prime} 0\right)=-2 .
$$

The exact solution is given by

$$
y(x)=(1-x) \sin x .
$$

The proposed method is tested on these three problems where the domain $[0,1]$ is divided into ten equal subintervals. The numerical results(absolute error) for the Example 5.1 are presented in Table $1-4$, due to the presented method along with the method discussed in [18]. In Table 16, maximum absolute error and maximum relative errors are mentioned at collocation points for different values of $J$. Figure 1, represents the physical behavior of numerical solution and exact solution for $J=6,7,8,9$ and $J=10$, respectively at grid points. Figure 4, represent the physical behavior of numerical solution and exact solution for $J=6,7,8,9$ and $J=10$ at collocation points. Similar explanations can be also given for Example 5.2 and Example 5.3. 


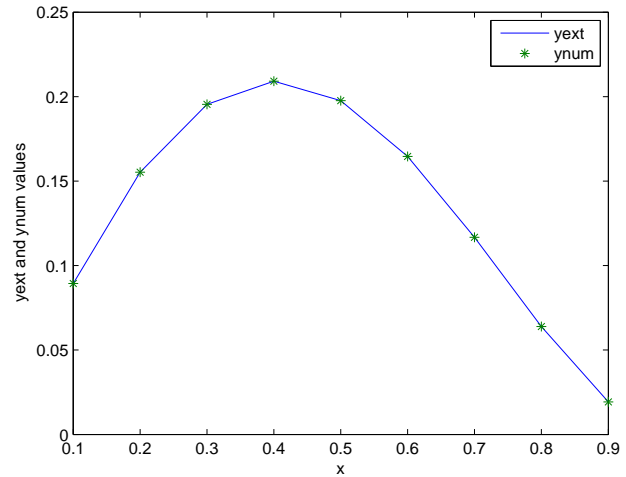

(a) $\mathrm{J}=6$

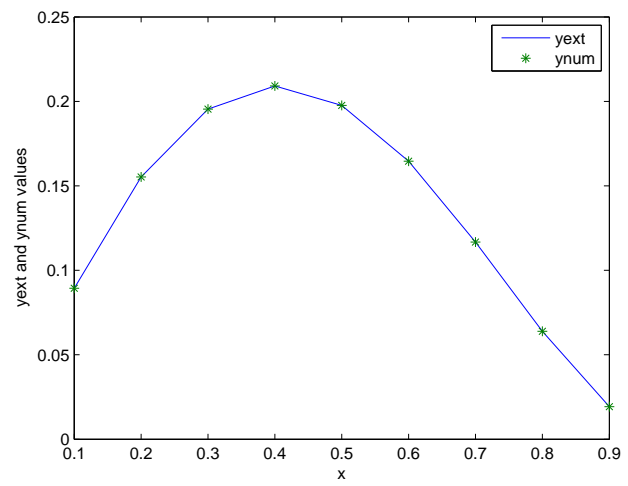

(c) $\mathrm{J}=8$

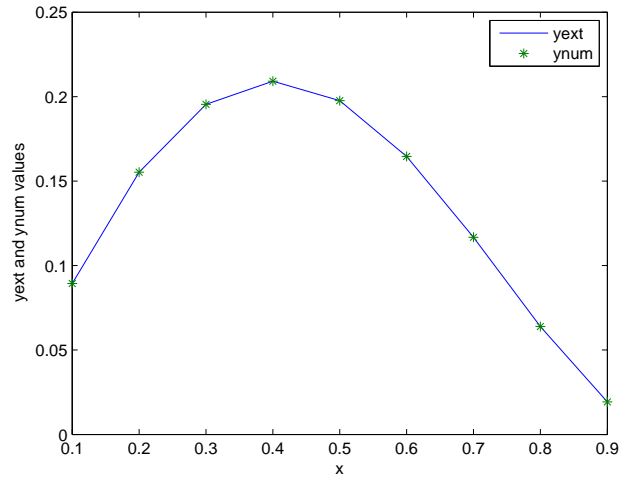

(b) $\mathrm{J}=7$

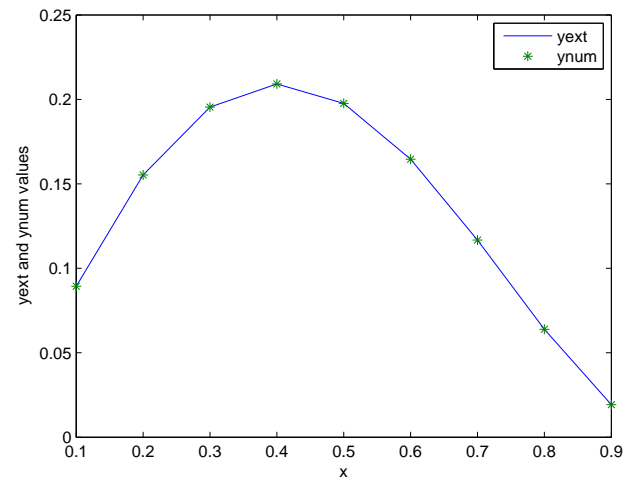

(d) $\mathrm{J}=9$

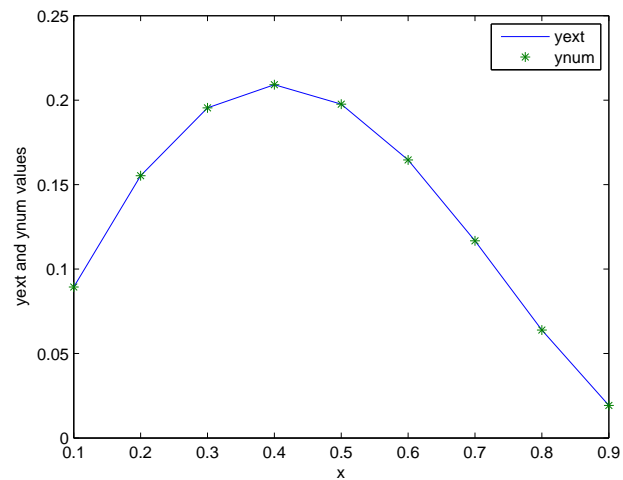

(e) $\mathrm{J}=10$

Figure 1: Physical behavior of exact and numerical solutions at grid points for Example 5.1 with different value of $J$ 


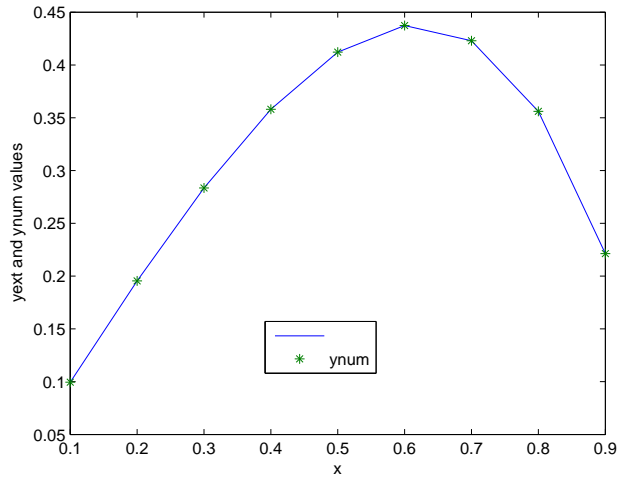

(a) $\mathrm{J}=6$

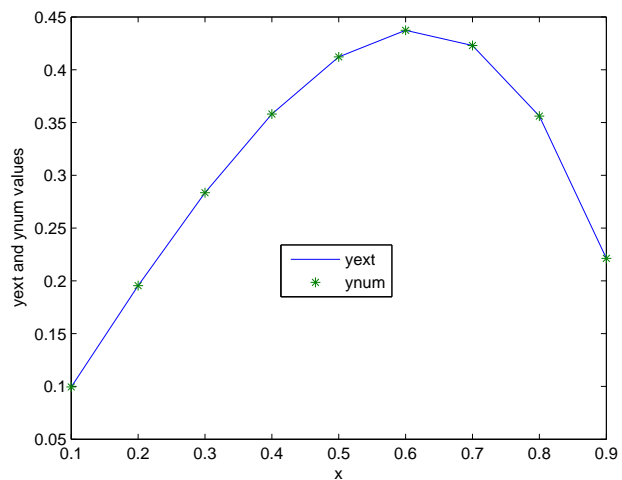

(c) $\mathrm{J}=8$

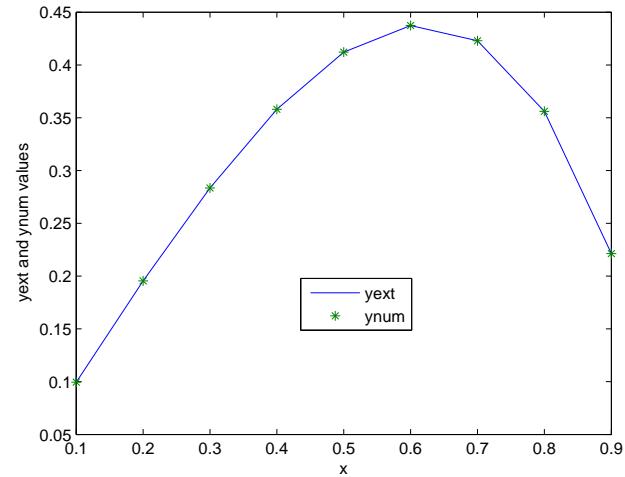

(b) $\mathrm{J}=7$

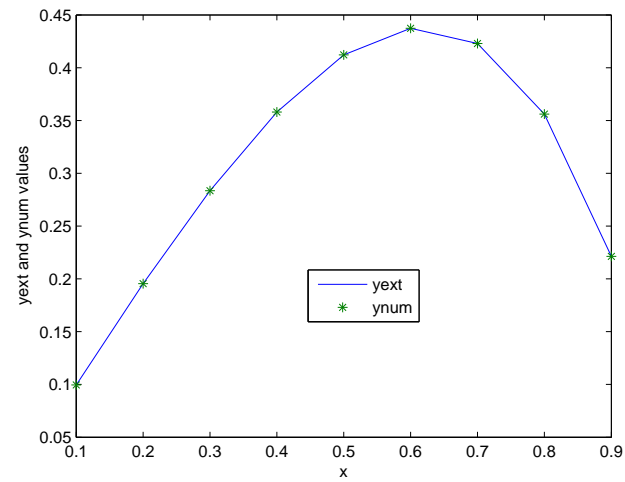

(d) $\mathrm{J}=9$

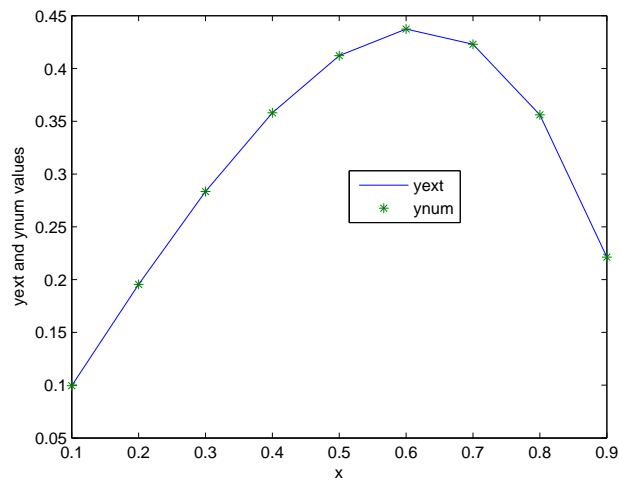

(e) $\mathrm{J}=10$

Figure 2: Physical behavior of exact and numerical solutions at grid points for Example 5.2 with different value of $J$ 


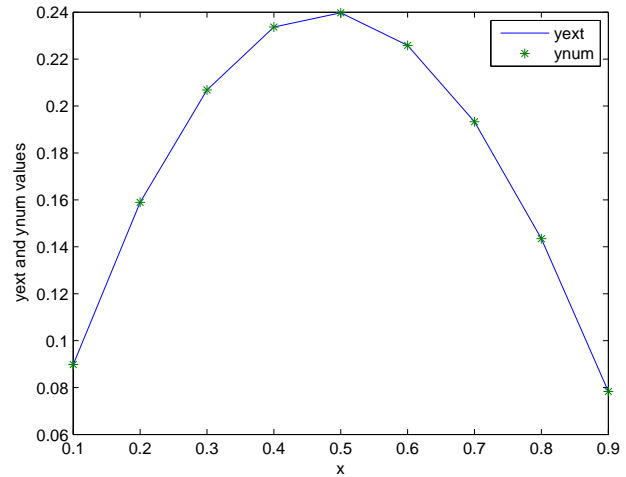

(a) $\mathrm{J}=6$

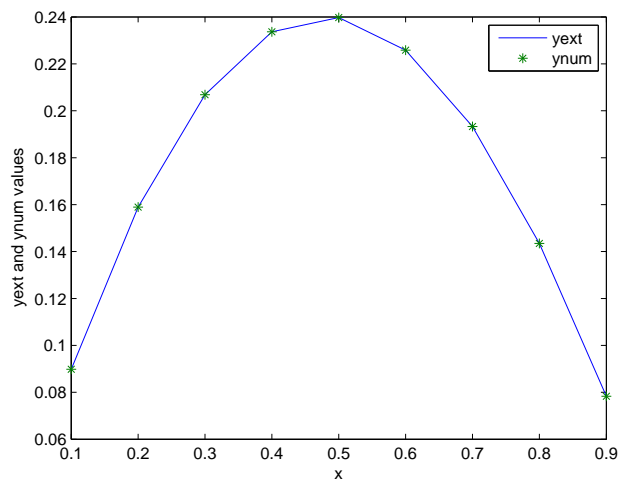

(c) $\mathrm{J}=8$

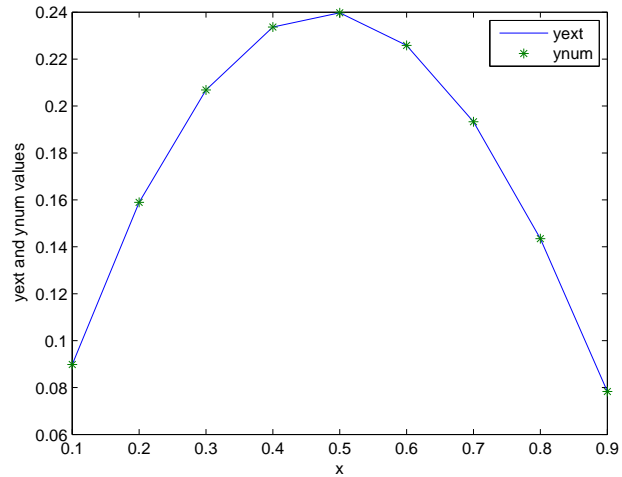

(b) $\mathrm{J}=7$

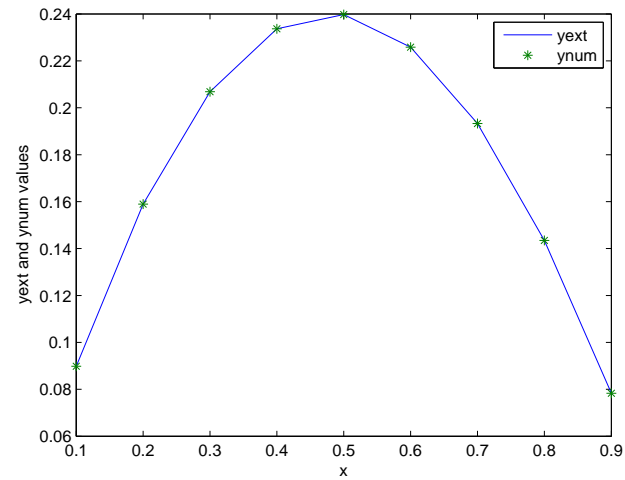

(d) $\mathrm{J}=9$

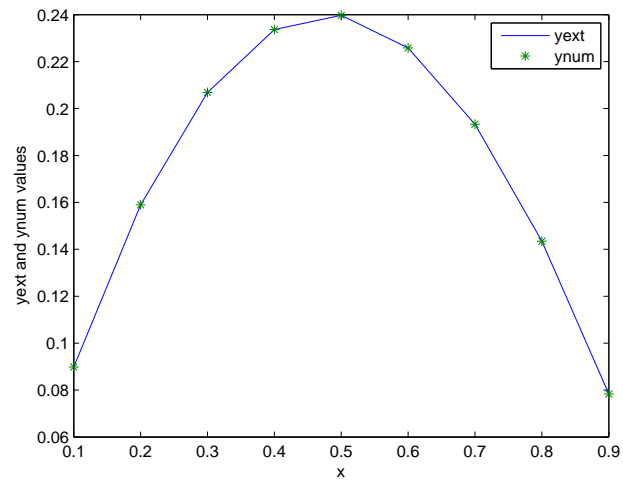

(e) $\mathrm{J}=10$

Figure 3: Physical behavior of exact and numerical solutions at grid points for Example 5.3 with different value of $J$ 


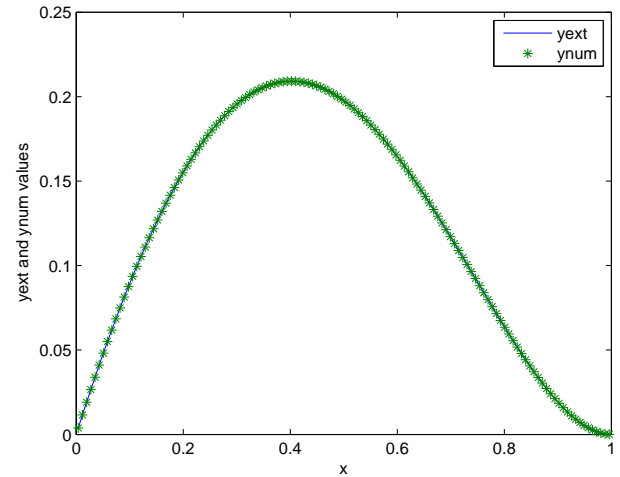

(a) $\mathrm{J}=6$

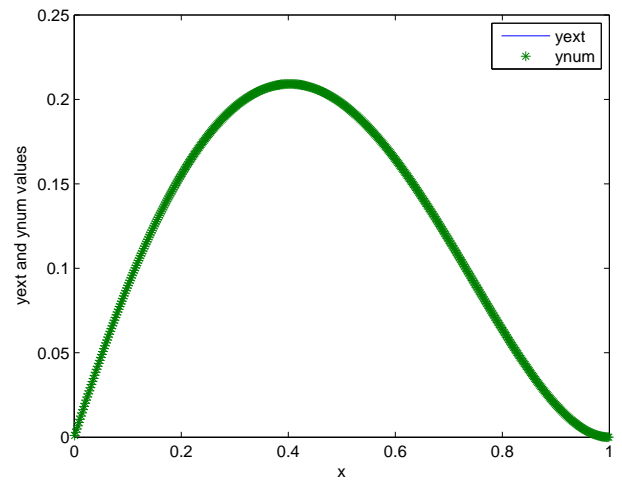

(c) $\mathrm{J}=8$

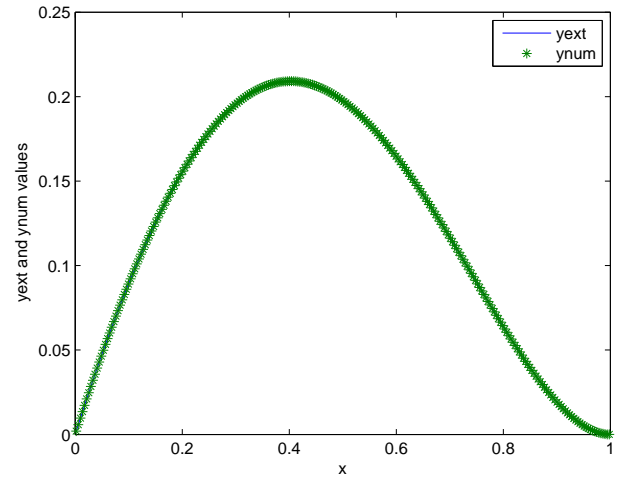

(b) $\mathrm{J}=7$

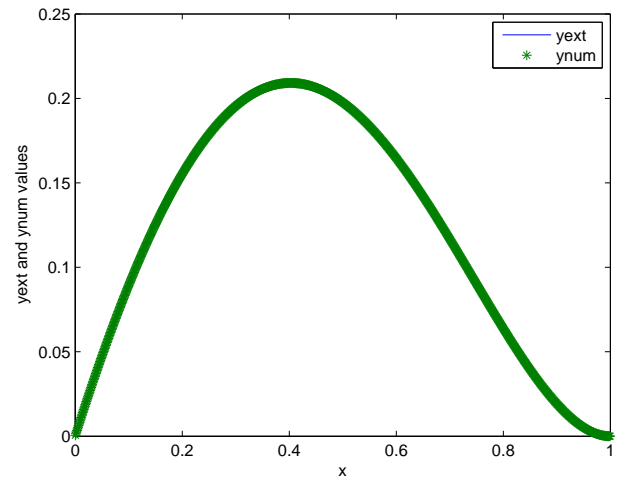

(d) $\mathrm{J}=9$

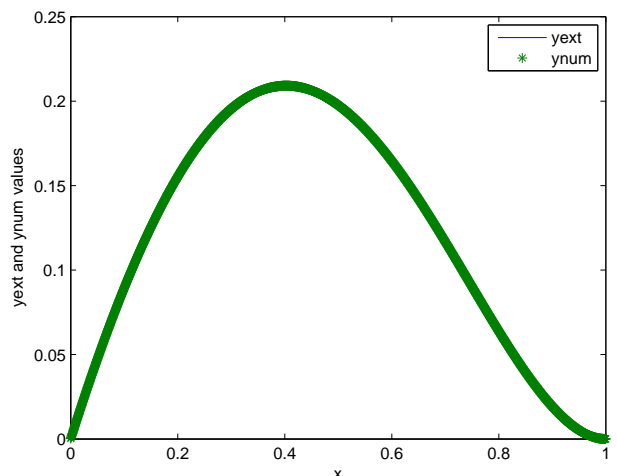

(e) $\mathrm{J}=10$

Figure 4: Physical behavior of exact and numerical solutions at collocation points for Example 5.1 with different value of $J$ 


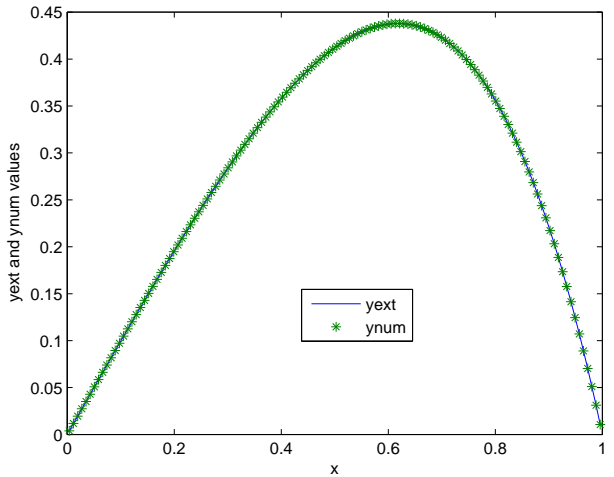

(a) $\mathrm{J}=6$

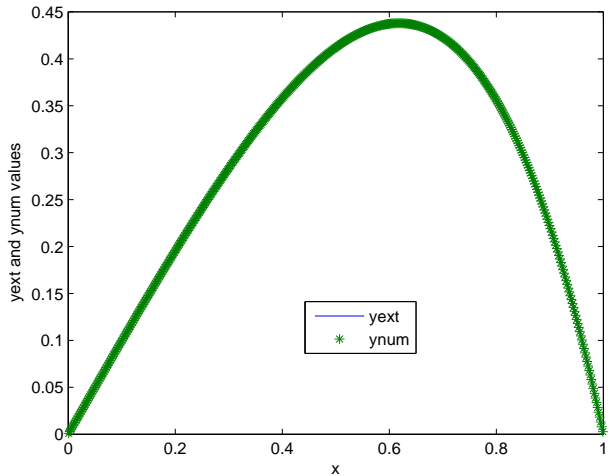

(c) $\mathrm{J}=8$

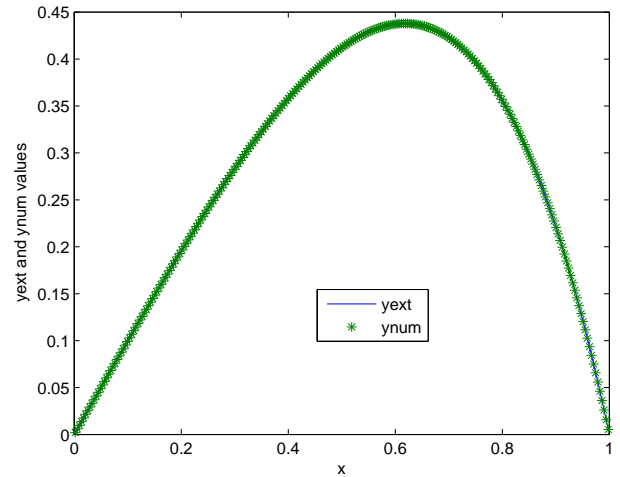

(b) $\mathrm{J}=7$

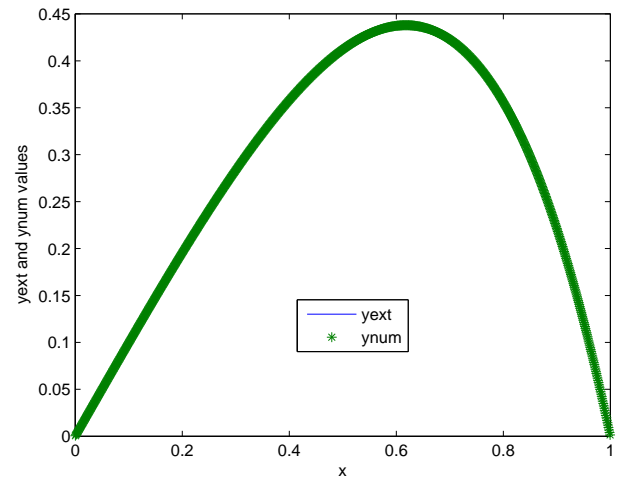

(d) $\mathrm{J}=9$

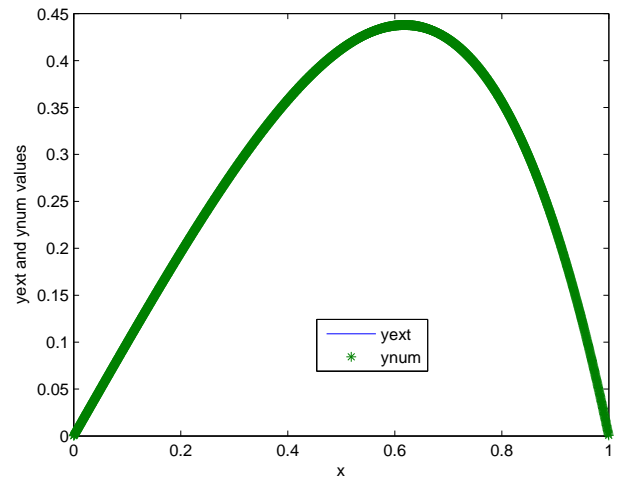

(e) $\mathrm{J}=10$

Figure 5: Physical behavior of exact and numerical solutions at collocation points for Example 5.2 with different value of $J$ 


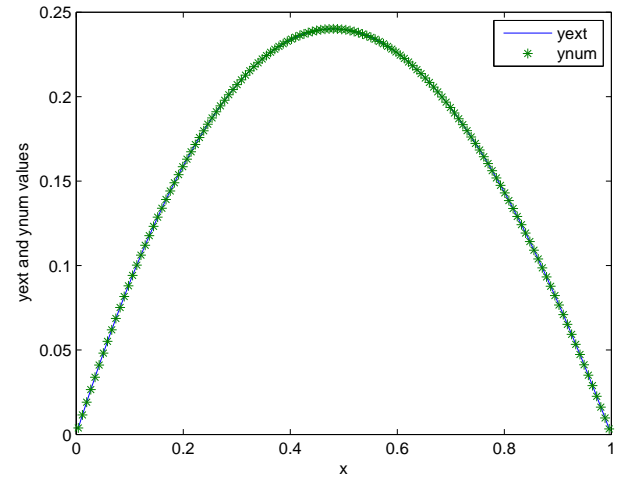

(a) $\mathrm{J}=6$

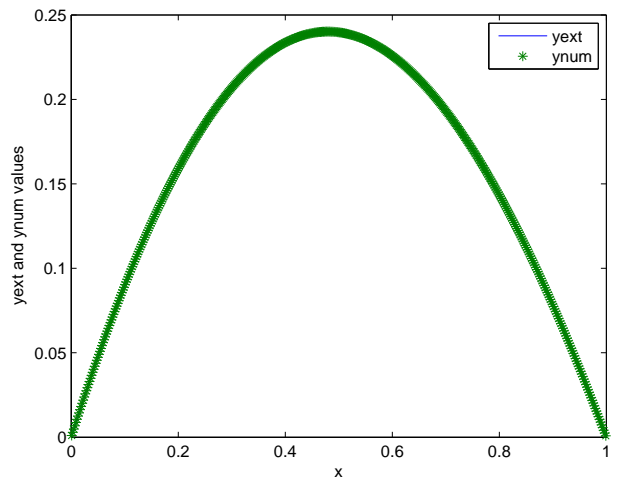

(c) $\mathrm{J}=8$

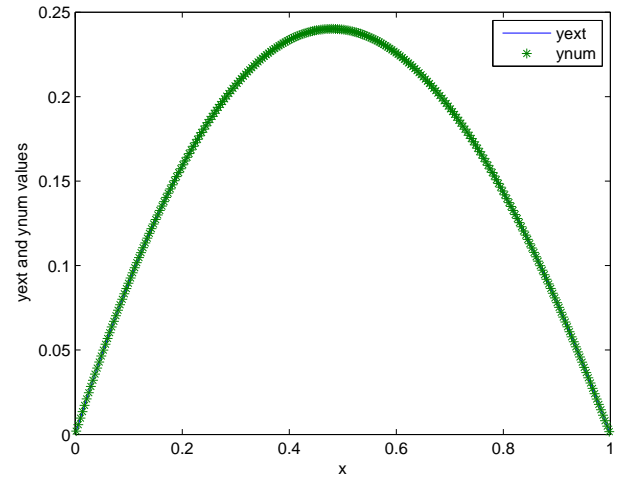

(b) $\mathrm{J}=7$

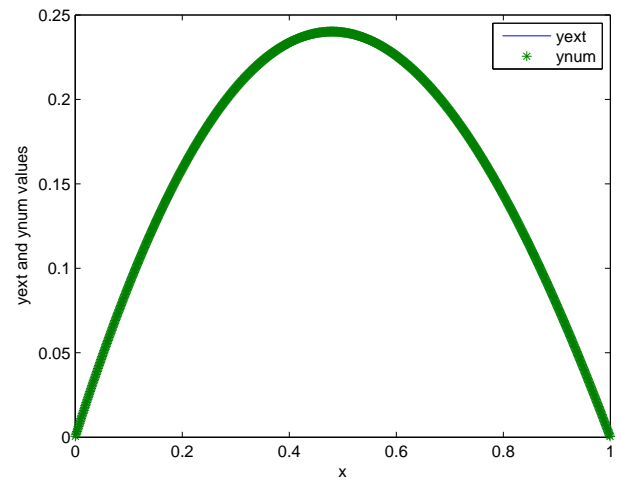

(d) $\mathrm{J}=9$

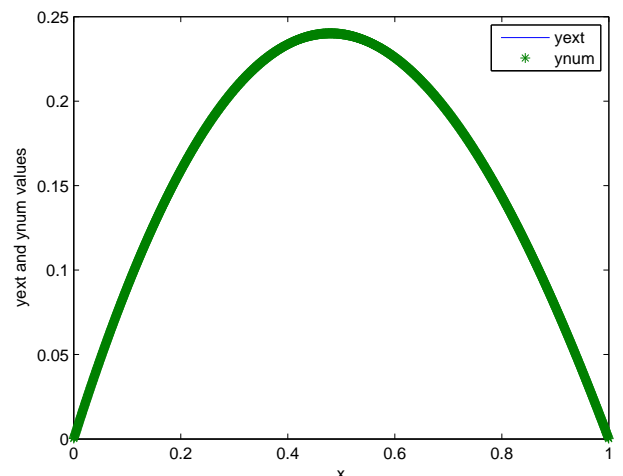

(e) $\mathrm{J}=10$

Figure 6: Physical behavior of exact and numerical solutions at collocation points for Example 5.3 with different value of $J$ 
Table 1: Comparison of the absolute error for Example 5.1 for $J=7$

\begin{tabular}{|c|c|c|}
\hline$x$ & Kashi et al.[18] & Present method \\
\hline 0.1 & $1.490116 \mathrm{E}-08$ & $9.426941 \mathrm{E}-10$ \\
0.2 & $1.788139 \mathrm{E}-07$ & $6.187677 \mathrm{E}-09$ \\
0.3 & $6.854534 \mathrm{E}-07$ & $1.659904 \mathrm{E}-08$ \\
0.4 & $7.599592 \mathrm{E}-07$ & $3.000117 \mathrm{E}-08$ \\
0.5 & $8.195639 \mathrm{E}-07$ & $4.221669 \mathrm{E}-08$ \\
0.6 & $8.344650 \mathrm{E}-07$ & $4.841840 \mathrm{E}-08$ \\
0.7 & $7.227063 \mathrm{E}-07$ & $4.483096 \mathrm{E}-08$ \\
0.8 & $4.321337 \mathrm{E}-07$ & $3.081452 \mathrm{E}-08$ \\
0.9 & $1.229346 \mathrm{E}-07$ & $1.135777 \mathrm{E}-08$ \\
\hline
\end{tabular}

Table 2: Comparison of the absolute error for Example 5.1 for $J=8$

\begin{tabular}{|c|c|c|}
\hline$x$ & Kashi et al.[18] & Present method \\
\hline 0.1 & $1.490116 \mathrm{E}-08$ & $2.356636 \mathrm{E}-10$ \\
0.2 & $1.788139 \mathrm{E}-07$ & $1.546888 \mathrm{E}-09$ \\
0.3 & $6.854534 \mathrm{E}-07$ & $4.149704 \mathrm{E}-09$ \\
0.4 & $7.599592 \mathrm{E}-07$ & $7.500221 \mathrm{E}-09$ \\
0.5 & $8.195639 \mathrm{E}-07$ & $1.055409 \mathrm{E}-08$ \\
0.6 & $8.344650 \mathrm{E}-07$ & $1.210453 \mathrm{E}-08$ \\
0.7 & $7.227063 \mathrm{E}-07$ & $1.120768 \mathrm{E}-08$ \\
0.8 & $4.321337 \mathrm{E}-07$ & $7.703598 \mathrm{E}-09$ \\
0.9 & $1.229346 \mathrm{E}-07$ & $2.839432 \mathrm{E}-09$ \\
\hline
\end{tabular}

Table 3: Comparison of the absolute error for Example 5.1 for $J=9$

\begin{tabular}{|c|c|c|}
\hline$x$ & Kashi et al.[18] & Present method \\
\hline 0.1 & $1.490116 \mathrm{E}-08$ & $5.891529 \mathrm{E}-11$ \\
0.2 & $1.788139 \mathrm{E}-07$ & $3.867200 \mathrm{E}-10$ \\
0.3 & $6.854534 \mathrm{E}-07$ & $1.037423 \mathrm{E}-09$ \\
0.4 & $7.599592 \mathrm{E}-07$ & $1.875051 \mathrm{E}-09$ \\
0.5 & $8.195639 \mathrm{E}-07$ & $2.638518 \mathrm{E}-09$ \\
0.6 & $8.344650 \mathrm{E}-07$ & $3.026127 \mathrm{E}-09$ \\
0.7 & $7.227063 \mathrm{E}-07$ & $2.801917 \mathrm{E}-09$ \\
0.8 & $4.321337 \mathrm{E}-07$ & $1.925898 \mathrm{E}-09$ \\
0.9 & $1.229346 \mathrm{E}-07$ & $7.098574 \mathrm{E}-10$ \\
\hline
\end{tabular}


Table 4: Comparison of the absolute error for Example 5.1 for $J=10$

\begin{tabular}{|c|c|c|}
\hline$x$ & Kashi et al.[18] & Present method \\
\hline 0.1 & $1.490116 \mathrm{E}-08$ & $1.472879 \mathrm{E}-11$ \\
0.2 & $1.788139 \mathrm{E}-07$ & $9.667991 \mathrm{E}-11$ \\
0.3 & $6.854534 \mathrm{E}-07$ & $2.593554 \mathrm{E}-10$ \\
0.4 & $7.599592 \mathrm{E}-07$ & $4.687624 \mathrm{E}-10$ \\
0.5 & $8.195639 \mathrm{E}-07$ & $6.596293 \mathrm{E}-10$ \\
0.6 & $8.344650 \mathrm{E}-07$ & $7.565315 \mathrm{E}-10$ \\
0.7 & $7.227063 \mathrm{E}-07$ & $7.004790 \mathrm{E}-10$ \\
0.8 & $4.321337 \mathrm{E}-07$ & $4.814744 \mathrm{E}-10$ \\
0.9 & $1.229346 \mathrm{E}-07$ & $1.774644 \mathrm{E}-10$ \\
\hline
\end{tabular}

Table 5: Comparison of the absolute error for Example 5.2 for $J=7$

\begin{tabular}{|c|c|c|}
\hline$x$ & Kashi et al.[18] & Present method \\
\hline 0.1 & $1.490116 \mathrm{E}-08$ & $3.975448 \mathrm{E}-10$ \\
0.2 & $1.490116 \mathrm{E}-08$ & $2.611241 \mathrm{E}-09$ \\
0.3 & $1.788139 \mathrm{E}-07$ & $7.014266 \mathrm{E}-09$ \\
0.4 & $6.258488 \mathrm{E}-07$ & $1.270369 \mathrm{E}-08$ \\
0.5 & $1.221895 \mathrm{E}-06$ & $1.792692 \mathrm{E}-08$ \\
0.6 & $1.251698 \mathrm{E}-06$ & $2.063586 \mathrm{E}-08$ \\
0.7 & $6.854534 \mathrm{E}-07$ & $1.919399 \mathrm{E}-08$ \\
0.8 & $2.384186 \mathrm{E}-07$ & $1.326558 \mathrm{E}-08$ \\
0.9 & $1.043081 \mathrm{E}-07$ & $4.921316 \mathrm{E}-09$ \\
\hline
\end{tabular}

Table 6: Comparison of the absolute error for Example 5.2 for $J=8$

\begin{tabular}{|c|c|c|}
\hline$x$ & Kashi et al.[18] & Present method \\
\hline 0.1 & $1.490116 \mathrm{E}-08$ & $9.939201 \mathrm{E}-11$ \\
0.2 & $1.490116 \mathrm{E}-08$ & $6.528284 \mathrm{E}-10$ \\
0.3 & $1.788139 \mathrm{E}-07$ & $1.753597 \mathrm{E}-09$ \\
0.4 & $6.258488 \mathrm{E}-07$ & $3.175962 \mathrm{E}-09$ \\
0.5 & $1.221895 \mathrm{E}-06$ & $4.481771 \mathrm{E}-09$ \\
0.6 & $1.251698 \mathrm{E}-06$ & $5.159001 \mathrm{E}-09$ \\
0.7 & $6.854534 \mathrm{E}-07$ & $4.798524 \mathrm{E}-09$ \\
0.8 & $2.384186 \mathrm{E}-07$ & $3.316411 \mathrm{E}-09$ \\
0.9 & $1.043081 \mathrm{E}-07$ & $1.230334 \mathrm{E}-09$ \\
\hline
\end{tabular}


Table 7: Comparison of the absolute error for Example 5.2 for $J=9$

\begin{tabular}{|c|c|c|}
\hline$x$ & Kashi et al.[18] & Present method \\
\hline 0.1 & $1.490116 \mathrm{E}-08$ & $2.484836 \mathrm{E}-11$ \\
0.2 & $1.490116 \mathrm{E}-08$ & $1.632083 \mathrm{E}-10$ \\
0.3 & $1.788139 \mathrm{E}-07$ & $4.384012 \mathrm{E}-10$ \\
0.4 & $6.258488 \mathrm{E}-07$ & $7.939930 \mathrm{E}-10$ \\
0.5 & $1.221895 \mathrm{E}-06$ & $1.120445 \mathrm{E}-09$ \\
0.6 & $1.251698 \mathrm{E}-06$ & $1.289753 \mathrm{E}-09$ \\
0.7 & $6.854534 \mathrm{E}-07$ & $1.199633 \mathrm{E}-09$ \\
0.8 & $2.384186 \mathrm{E}-07$ & $8.291039 \mathrm{E}-10$ \\
0.9 & $1.043081 \mathrm{E}-07$ & $3.075838 \mathrm{E}-10$ \\
\hline
\end{tabular}

Table 8: Comparison of the absolute error for Example 5.2 for $J=10$

\begin{tabular}{|c|c|c|}
\hline$x$ & Kashi et al.[18] & Present method \\
\hline 0.1 & $1.490116 \mathrm{E}-08$ & $6.212114 \mathrm{E}-12$ \\
0.2 & $1.490116 \mathrm{E}-08$ & $4.080217 \mathrm{E}-11$ \\
0.3 & $1.788139 \mathrm{E}-07$ & $1.096004 \mathrm{E}-10$ \\
0.4 & $6.258488 \mathrm{E}-07$ & $1.984984 \mathrm{E}-10$ \\
0.5 & $1.221895 \mathrm{E}-06$ & $2.801114 \mathrm{E}-10$ \\
0.6 & $1.251698 \mathrm{E}-06$ & $3.224382 \mathrm{E}-10$ \\
0.7 & $6.854534 \mathrm{E}-07$ & $2.999082 \mathrm{E}-10$ \\
0.8 & $2.384186 \mathrm{E}-07$ & $2.072761 \mathrm{E}-10$ \\
0.9 & $1.043081 \mathrm{E}-07$ & $7.689613 \mathrm{E}-11$ \\
\hline
\end{tabular}

Table 9: Comparison of the absolute error for Example 5.3 for $J=7$

\begin{tabular}{|c|c|c|}
\hline$x$ & Kashi et al.[18] & Present method \\
\hline 0.1 & $7.450581 \mathrm{E}-09$ & $7.511505 \mathrm{E}-12$ \\
0.2 & $7.450581 \mathrm{E}-08$ & $5.092640 \mathrm{E}-11$ \\
0.3 & $3.278255 \mathrm{E}-07$ & $1.404676 \mathrm{E}-10$ \\
0.4 & 0.0000000000 & $2.599481 \mathrm{E}-10$ \\
0.5 & $2.831221 \mathrm{E}-07$ & $3.731419 \mathrm{E}-10$ \\
0.6 & $3.427267 \mathrm{E}-07$ & $4.351287 \mathrm{E}-10$ \\
0.7 & $1.490116 \mathrm{E}-07$ & $4.084567 \mathrm{E}-10$ \\
0.8 & $4.470348 \mathrm{E}-08$ & $2.839061 \mathrm{E}-10$ \\
0.9 & $2.235174 \mathrm{E}-08$ & $1.055810 \mathrm{E}-10$ \\
\hline
\end{tabular}


Table 10: Comparison of the absolute error for Example 5.3 for $J=8$

\begin{tabular}{|c|c|c|}
\hline$x$ & Kashi et al.[18] & Present method \\
\hline 0.1 & $7.450581 \mathrm{E}-09$ & $1.876416 \mathrm{E}-12$ \\
0.2 & $7.450581 \mathrm{E}-08$ & $1.272693 \mathrm{E}-11$ \\
0.3 & $3.278255 \mathrm{E}-07$ & $3.510892 \mathrm{E}-11$ \\
0.4 & 0.0000000000 & $6.497655 \mathrm{E}-11$ \\
0.5 & $2.831221 \mathrm{E}-07$ & $9.327414 \mathrm{E}-11$ \\
0.6 & $3.427267 \mathrm{E}-07$ & $1.087717 \mathrm{E}-10$ \\
0.7 & $1.490116 \mathrm{E}-07$ & $1.021062 \mathrm{E}-10$ \\
0.8 & $4.470348 \mathrm{E}-08$ & $7.097176 \mathrm{E}-11$ \\
0.9 & $2.235174 \mathrm{E}-08$ & $2.639368 \mathrm{E}-11$ \\
\hline
\end{tabular}

Table 11: Comparison of the absolute error for Example 5.3 for $J=9$

\begin{tabular}{|c|c|c|}
\hline$x$ & Kashi et al.[18] & Present method \\
\hline 0.1 & $7.450581 \mathrm{E}-09$ & $4.690276 \mathrm{E}-13$ \\
0.2 & $7.450581 \mathrm{E}-08$ & $3.181427 \mathrm{E}-12$ \\
0.3 & $3.278255 \mathrm{E}-07$ & $8.776757 \mathrm{E}-12$ \\
0.4 & 0.0000000000 & $1.624348 \mathrm{E}-11$ \\
0.5 & $2.831221 \mathrm{E}-07$ & $2.331782 \mathrm{E}-11$ \\
0.6 & $3.427267 \mathrm{E}-07$ & $2.719228 \mathrm{E}-11$ \\
0.7 & $1.490116 \mathrm{E}-07$ & $2.552608 \mathrm{E}-11$ \\
0.8 & $4.470348 \mathrm{E}-08$ & $1.774250 \mathrm{E}-11$ \\
0.9 & $2.235174 \mathrm{E}-08$ & $6.598236 \mathrm{E}-12$ \\
\hline
\end{tabular}

Table 12: Comparison of the absolute error for Example 5.3 for $J=10$

\begin{tabular}{|c|c|c|}
\hline$x$ & Kashi et al.[18] & Present method \\
\hline 0.1 & $7.450581 \mathrm{E}-09$ & $1.172673 \mathrm{E}-13$ \\
0.2 & $7.450581 \mathrm{E}-08$ & $7.953083 \mathrm{E}-13$ \\
0.3 & $3.278255 \mathrm{E}-07$ & $2.194162 \mathrm{E}-12$ \\
0.4 & 0.0000000000 & $4.060835 \mathrm{E}-12$ \\
0.5 & $2.831221 \mathrm{E}-07$ & $5.829420 \mathrm{E}-12$ \\
0.6 & $3.427267 \mathrm{E}-07$ & $6.798034 \mathrm{E}-12$ \\
0.7 & $1.490116 \mathrm{E}-07$ & $6.381534 \mathrm{E}-12$ \\
0.8 & $4.470348 \mathrm{E}-08$ & $4.435480 \mathrm{E}-12$ \\
0.9 & $2.235174 \mathrm{E}-08$ & $1.649458 \mathrm{E}-12$ \\
\hline
\end{tabular}


Table 13: Maximum absolute error and Maximum relative error for Example 5.1

\begin{tabular}{|c|c|c|c|}
\hline$J$ & $M$ & Maximum absolute error & Maximum relative error \\
\hline 1 & 4 & $2.088749 \mathrm{E}-04$ & $1.003371 \mathrm{E}-03$ \\
2 & 8 & $4.869002 \mathrm{E}-05$ & $2.345076 \mathrm{E}-04$ \\
3 & 16 & $1.238865 \mathrm{E}-05$ & $5.923845 \mathrm{E}-05$ \\
4 & 32 & $3.107995 \mathrm{E}-06$ & $1.487326 \mathrm{E}-05$ \\
5 & 64 & $7.771911 \mathrm{E}-07$ & $3.716372 \mathrm{E}-06$ \\
6 & 128 & $1.942499 \mathrm{E}-07$ & $9.287656 \mathrm{E}-07$ \\
7 & 256 & $4.856704 \mathrm{E}-08$ & $2.322170 \mathrm{E}-07$ \\
8 & 512 & $1.214203 \mathrm{E}-08$ & $5.805473 \mathrm{E}-08$ \\
9 & 1024 & $3.035522 \mathrm{E}-09$ & $1.451372 \mathrm{E}-08$ \\
10 & 2048 & $7.588812 \mathrm{E}-10$ & $3.628431 \mathrm{E}-09$ \\
\hline
\end{tabular}

Table 14: Maximum absolute error and Maximum relative error for Example 5.2

\begin{tabular}{|c|c|c|c|}
\hline$J$ & $M$ & Maximum absolute error & Maximum relative error \\
\hline 1 & 4 & $8.285245 \mathrm{E}-05$ & $1.892169 \mathrm{E}-04$ \\
2 & 8 & $2.031412 \mathrm{E}-05$ & $4.703345 \mathrm{E}-05$ \\
3 & 16 & $5.254683 \mathrm{E}-06$ & $1.203060 \mathrm{E}-05$ \\
4 & 32 & $1.323702 \mathrm{E}-06$ & $3.023412 \mathrm{E}-06$ \\
5 & 64 & $3.313914 \mathrm{E}-07$ & $7.566533 \mathrm{E}-07$ \\
6 & 128 & $8.285614 \mathrm{E}-08$ & $1.891900 \mathrm{E}-07$ \\
7 & 256 & $2.071555 \mathrm{E}-08$ & $4.7299122 \mathrm{E}-08$ \\
8 & 512 & $5.178881 \mathrm{E}-09$ & $1.1824700 \mathrm{E}-08$ \\
9 & 1024 & $1.294731 \mathrm{E}-09$ & $2.956201 \mathrm{E}-09$ \\
10 & 2048 & $3.236832 \mathrm{E}-10$ & $7.390509 \mathrm{E}-10$ \\
\hline
\end{tabular}

Table 15: Maximum absolute error and Maximum relative error for Example 5.3

\begin{tabular}{|c|c|c|c|}
\hline$J$ & $M$ & Maximum absolute error & Maximum relative error \\
\hline 1 & 4 & $2.650027 \mathrm{E}-06$ & $1.157620 \mathrm{E}-05$ \\
2 & 8 & $4.915768 \mathrm{E}-07$ & $2.062693 \mathrm{E}-06$ \\
3 & 16 & $1.146593 \mathrm{E}-07$ & $4.777401 \mathrm{E}-07$ \\
4 & 32 & $2.817731 \mathrm{E}-08$ & $1.173548 \mathrm{E}-07$ \\
5 & 64 & $7.014512 \mathrm{E}-09$ & $2.921312 \mathrm{E}-08$ \\
6 & 128 & $1.751717 \mathrm{E}-09$ & $7.295031 \mathrm{E}-09$ \\
7 & 256 & $4.3779935 \mathrm{E}-10$ & $1.823224 \mathrm{E}-09$ \\
8 & 512 & $1.094402 \mathrm{E}-10$ & $4.557631 \mathrm{E}-10$ \\
9 & 1024 & $2.735945 \mathrm{E}-11$ & $1.139383 \mathrm{E}-10$ \\
10 & 2048 & $6.839862 \mathrm{E}-12$ & $2.848456 \mathrm{E}-11$ \\
\hline
\end{tabular}




\section{CONCLUSION}

In the present article, we have presented a numerical solution of fifth-order boundary value problems using Haar wavelets. The proposed method has been tested on three fifth order boundary value problems. The numerical results obtained by the proposed method are in good agreement with the exact solutions available in the literature. The objective of this paper is to present a simple and efficient method to solve a general fifth order linear boundary value problem.

\section{REFERENCES}

[1] Caglar, H. N., Caglar, S. H. and Trizell, E. H., (1999), The numerical solution of fifth-order boundary value problems with sixth-degree B-spline functions, Appl. Math. Lett. 12, pp. 25-30.

[2] Davies, A. R., Karageorghis, A. and Phillips, T. N., (1998), Spectral Galerkin method for the primary two point boundary value problem in modelling viscoelastic flows, Int. J. Num. Meth. Engg. 26, pp. 647-662.

[3] Fyfe, D. J., (1974), Linear dependence relations connecting equal interval $N^{t h}$ degree spline and their derivatives, J. Inst. Math. Appl., 7, pp. 398-406.

[4] Noor, M. A. and Mohyud-Din, S.T., (2007), An efficient algorithm for solving fifth-order boundary value problems, Math. Com. Mod. , 45, pp. 954-964.

[5] Noor, M. A. and Mohyud-Din S.T., (2007), Variational iteration technique for solving higher order boundary value problems, App. Math. Comput., 189, pp. 1929-1942.

[6] Noor, M. A. and Mohyud-Din S.T., : Modified decomposition method for solving linear and nonlinear fifth-order boundary value problems, Int. J. Appl. Math. Comp. Sci. In press.

[7] Wazwaz, A. M., (2001), The numerical solution of fifth-order boundary value problems by decomposition method, J. Comp. Appl. Math., 136, pp. 259-270.

[8] Viswanadham, K. N. S. K. and Kiranmayi, S. V., (2017), Numerical solution of fifth order boundary value problems by petrov-Galerkin method with quartic B-spline as basis functions and Sextic B-spline as weight functions, Int. J. Comp. Appl., 161(10), pp. 0975 - 8887.

[9] Agarwal, R. P., (1986), Boundary value problems for higher order differential equations. World Scientific, Singapore. 
[10] Hsiao, C. H., (2004), Haar wavelets approach to linear stiff systems, Maths. Comp. Simul., 64, pp. 561-567.

[11] Lepik, U., (2005), Numerical solution of differential equation using Haar wavelets, Maths. Comp. Simul., 68, pp. 127-143.

[12] Lepik, U., (2007), Numerical solution of evolution equation by Haar wavelets, Appl. Maths. Comp., 185, pp. 695-704.

[13] Kumar, M., and Pandit S., (2012), Wavelet transform and wavelet based numerical methods, Int. Jour. Nol. Scie. , 69, 31-37.

[14] Haq, F., Hussain, I. and Ali, A., (2011), A Haar wavelets based numerical method for third-order boundary and initial value problems, W. Appl. Scie. J., 10, pp. 2244-2251.

[15] Haq, F. I., (2011), The numerical solution of fourth order boundary-value using Haar wavelets, Appl. Math. Scie., 63, pp. 3131-3146.

[16] Haq, F. I., Ali, A. and Hussain I., (2012), Solution of sixth- order boundary-value problems by collocation method using Haar wavelets, Int. J. Phy. Sci. , 43, pp. 5729-5735.

[17] Babolian, E. and Shahsawaran,A., (2009), Numerical solution of nonlinear Fredholm integral equation of second kind using Haar wavelets, J. Comput. Apply. math., 225, pp. 87-95.

[18] Viswanadham, K. N. S. K. and Ballem, S., (2013), Numerical solution of fifth order boundary value problems by Galerkin Method with quartic B-splines, Int. J. Comp. Appl., 77(17), pp. 0975-8887. 
